\title{
Research on Fire Fighting Integrated Treatment System based on Internet of Things \\ Yingna Qiao
}

\author{
Benxi fire department of Liaoning, Liaoning 117000, China
}

Keywords: Internet of things, fire fighting, integrated treatment system, research and analysis.

\begin{abstract}
In recent years, the technology of Internet of things has been paid more and more attention, and its application has become the focus of attention. It is necessary to provide strong technical support to meet the needs of social and economic development. This paper briefly introduces the concept of networking technology, the actual needs of the development situation and fire forces with IOT of the feasibility analysis of networking technology in the field of fire protection application, and the application prospect of fire forces in fire fighting and rescue, fire safety management, fire protection equipment and social problems existing in the management are discussed in detail as network technology in the field of fire protection, pointed out that the application of this technology will play an important role in improving the level and efficiency of fire protection.
\end{abstract}

\section{Introduction}

The concept of things was first proposed in 1999 by means of radio frequency identification (RFID), infrared sensors, GPS, laser scanners and other information sensing equipment, in accordance with the contract agreement, will anything connected to the Internet, information exchange and communication, a network technology to realize intelligent identification, positioning, tracking, monitoring and management.

From the technical point of view, the Internet of things system can be divided into 3 layers, namely: the perception layer, network layer and application layer. According to the fire field, the perception layer refers to has fire sensing equipment, installed in a building alarm monitoring equipment and automatic fire extinguishing equipment, application layer refers to the fire department, fire department key, and network layer refers to the hidden dangers information perception layer transfer to the application layer through wired or wireless transmission, and application the fire command is transmitted to the perception layer.

The typical application of the Internet of things in the field of fire protection is the city fire remote monitoring system (FAS), the whole system consists of remote monitoring terminal remote monitoring communication network and remote monitoring center. In recent years, the phenomenon of fire in our country is becoming more and more serious. Especially within the city of large buildings, indoor fire personnel due to the characteristics of high density, high space, causes complex problems caused by the disaster losses and casualties, rescue difficult, rescue situation becomes more serious. The Internet of things technology has become a research hotspot at home and abroad, and the research on the application of the Internet of things technology in the field of indoor firefighting is also increasing. 


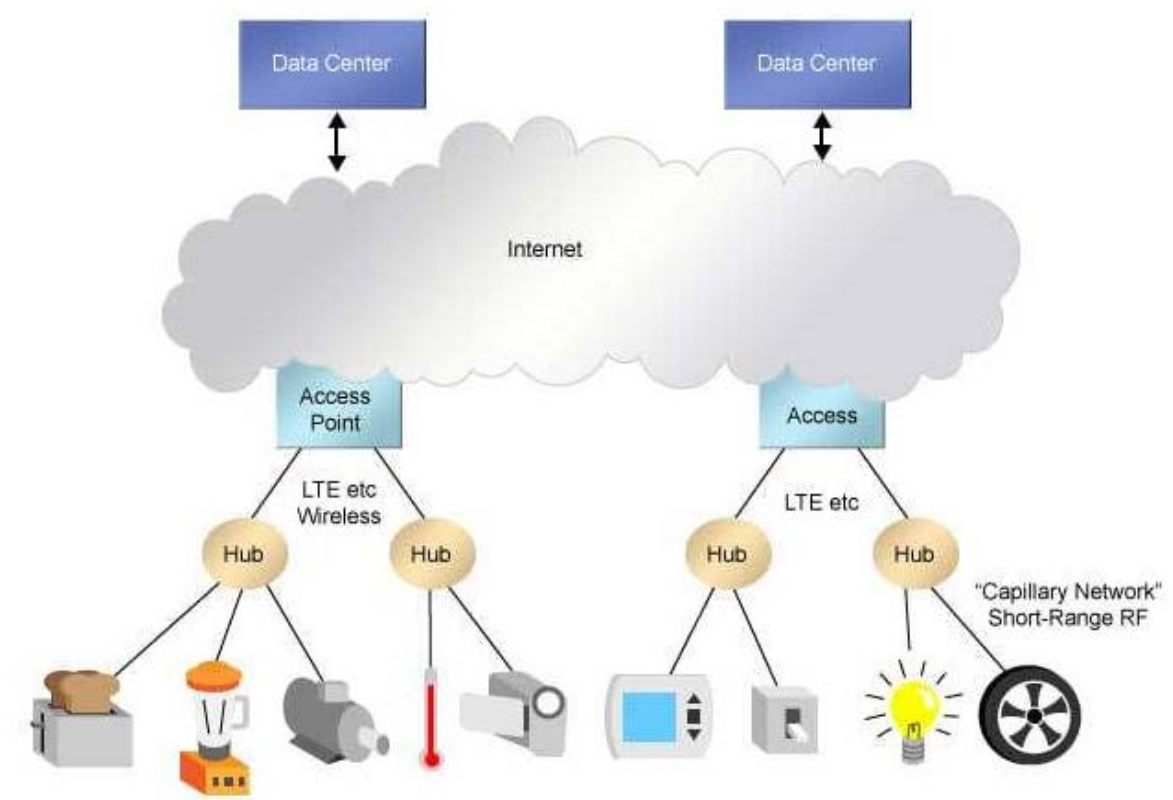

Figure.1 Internet of things

\section{The Proposed Methodology}

\subsection{Internet of things technology}

Through the radio frequency identification (RFID), infrared sensors, GPS, laser scanners and other information sensing equipment, in accordance with the contract agreement, to anything connected with the Internet, information exchange and communication, to achieve a network intelligent identification, positioning, tracking, monitoring and management. It can be seen from the concept of the Internet of things on the Internet is based on the extension and expansion of the network, which realizes the interaction between people and people, people and things, things and things.

The Internet of things is developed based on the existing information network, is the extension and expansion on the basis of the current communication network and the Internet, generally do not need to transform the most basic network of things. Two dimensional code, sensors, electronic tags and other sensing devices, the Internet, communications networks, sensor networks and other network facilities have been widely used in all walks of life, and the formation of a more mature application model. Therefore, the Internet of things technology used in the field of fire has a wealth of experience can learn from. In addition, since the implementation of fire information construction, wired communications, wireless communications, satellite communications, computer communications and other infrastructure communication network has been basically established in the fire brigade, the Internet technology and the practical system has been widely used in fire fighting forces. These provide the basic technical conditions for the application of the Internet of things.

At present, the key unit of fire safety have been put into use of automatic fire alarm system and other emergency management system, and links to the fire safety remote monitoring system over, these systems in the aspects of information collection, transmission, processing and networking technology have some similarities, as long as the re construction of a layer based on the IOT technology in the system based on this, you can use networking technology to effectively expand the existing emergency management system function. Fire brigade is currently equipped with the use of fire rescue command system, 3G image transmission system for the Internet of things provides a platform for the application.

\subsection{Fire safety management}

Low technical content led to the current work model can not meet the characteristics of the dynamic management of social fire safety, fire safety management and the actual needs of the contradiction between the prominent. The Internet of things technology provides an effective way for the innovation of fire safety management mode. For example, the use of RFID technology, the video 
monitoring system can real-time monitoring of key units in the fire control room personnel management, avoid staff off-site phenomenon; collecting operation state of building fire protection facilities and internal fixation using sensor technology, and through the server access network, remote control and management, so as to improve the effective fire supervision and management work.

In recent years, fire fighting and rescue tasks for the fire forces more and more arduous, facing the disaster scene more and more complex, often encountered in the implementation of the rescue scene is not clear, the transmission of information is not smooth, decision-making is not scientific problems. The Internet of things technology has a comprehensive perception, reliable delivery, intelligent processing of the three characteristics, can be a good solution to these problems, to meet the needs of the fire brigade to deal with all kinds of disasters. Wireless sensor network (WSN) can be used to detect the disaster scene, especially the harsh environment. The sensor technology for safety management of the fire fighting and rescue personnel, real-time vital signs parameters, the position information is transmitted to the firefighter's commanders; analysis of integration, the use of cloud computing technology to collect intelligence information, provides the support for the commander's decision, in order to improve scientific decision-making.

\subsection{Intelligent command}

In the implementation of fire rescue process, attack route selection, hose laying, water position setting, gun positions setting is the key for successful rescue, and smoke, heat, toxic fire scene is the main threat to the personal safety of fire fighters. After the emergence of the Internet of things, in the fire fighting and rescue equipment on embedded micro sensor chip, use of wireless communication networks, and the Internet to achieve seamless, gun head, water hose, pressure, position, and other subordinate units of real-time information uploaded to the command center, command center can be adjusted at any time according to the scene the deployment of the fire. Combat personnel entering the scene, with the help of various sensing devices, such as RFID devices, infrared sensors, global positioning system, toxic gas induction device, temperature and humidity sensors, combined with the Internet to form a huge network of things. According to the information of the network, the commander in charge of the rear command can control the temperature, the concentration of harmful gas, the pressure of the water gun and so on. In addition, the system can be used to collect the video and audio data, determine the location of firefighters, communicate with firefighters, improve the overall combat effectiveness of the troops, to protect the personal safety of combatants.

\subsection{Force management}

The use of networking technology, real-time control of army duty combat readiness order, duty monitoring, statistical analysis, quantitative force, can discover and solve the problem, improve the army management level of education, implementation of troops management standardization.

The use of networking technology, building intelligent system on duty combat readiness management, a central management platform, acquisition terminal, backbone terminal, vehicle terminal, identification tags and other parts, the whole system is based on wireless sensor network as the core, the use of GPS, wireless communication technology, to form a complete set of vehicles and personnel management system. The duty vehicles installed by the vehicle terminal GPS module, data acquisition module, wireless communication module, the acquisition module of vehicle equipment timely collection of label information, dynamic confirmation number and number and type of personnel onboard equipment, and then through the wireless network to the rear of the command center, the command center at any time convenient vehicle location, vehicle equipment the situation. The sensor chip is embedded in the fire fighting and rescue combat clothing, and the position, the distribution and the quantity of the combatants are transmitted to the rear command center through the wireless transmission mode, and the combat personnel are managed in real time.

The use of networking technology, different places and different types of fire material centralized, intelligent management, scattered equipment, reagents for information integration into a unified network, enhance the fire protection work and force development level. First of all, according to the type of material fire protection function, subordinate units and other attributes are classified, and then the RFID label in every kind of fire material, the use of RFID, the short distance wireless network technology, real-time data recording materials in the storage and transportation of materials, use of 
fire in the process, and the data in accordance with the squadron, Corps detachment, classification statistics. In this way, once the large-scale disaster occurs, intelligent equipment management system can dynamically display the use and inventory of fire materials. Once the rescue operation is completed, you can immediately count the number of materials consumed by the equipment, to provide scientific data for the fire summary.

With the help of Internet technology, through wireless or wired communication equipment installed in the fire hydrant, fire water, natural lakes and other important position sensor is triggered by flowing water, regular water will send the information to the central server by installing the SIM card mobile phone, PDA, wireless or wired computer terminal equipment, pressure state, real-time query of fire water, to achieve real-time network monitoring of the fire water. With the help of GPS, GIS and other communication equipment positioning information, timely and dynamic grasp of the various fire water source location information. Once the disaster accident, fire water can be real-time information through networking technology accurately transmitted to the command center and combat vehicles, and provide accurate information for the rapid expansion of water fire fighting and rescue operations.

\subsection{Remote management of building fire protection facilities}

Intelligent video traffic statistics, is to achieve a kind of technology of the passenger flow statistics analysis using intelligent video technology, common methods are divided into three types: binocular stereo vision based passenger flow estimation based on the principle of binocular disparity, traffic statistics technology of moving target detection and recognition of video based pattern matching technology and motion tracking technology based on. Through intelligent video traffic statistics technology, can provide effective traffic monitoring and guiding basis, and the total population of the region, the flow of personnel and personnel density trends in real-time monitoring, once in case of fire and other emergency situations, to scientifically crowd grooming, the major activities can ensure a safe and orderly manner. The specific principle is: according to the real-time collection of user location information, the system defines the number of users in the region, and the definition of the region to provide real-time mobile passenger flow statistics and historical passenger flow statistics.

\section{Conclusion}

With the continuous development and maturity of the Internet of things technology, the application of Internet of things in all walks of life will continue to expand and deepen. Bring about profound changes in its application in the fire field will be on the development of the cause of the fire, to promote the major changes in fire forces in the fire rescue, fire supervision management, logistics management and other aspects, effectively improve the fire fighting rescue and fire supervision and law enforcement ability. Therefore, we should grasp the development of networking technology which brings opportunities.

\section{References}

[1] Yang, Shuang-Hua. "Internet of things."Wireless Sensor Networks. Springer London, 2014. 247-261.

[2] Perera, Charith, et al. "Context aware computing for the internet of things: A survey." IEEE Communications Surveys \& Tutorials 16.1 (2014): 414-454.

[3] Wang, H. and Wang, J., 2014, November. An effective image representation method using kernel classification. In Tools with Artificial Intelligence (ICTAI), 2014 IEEE 26th International Conference on (pp. 853-858). IEEE.

[4] Raza, Shahid, et al. "Lithe: Lightweight secure CoAP for the internet of things." IEEE Sensors Journal 13.10 (2013): 3711-3720.

[5] Jan, Mian Ahmad, et al. "A robust authentication scheme for observing resources in the internet of things environment." Trust, Security and Privacy in Computing and Communications (TrustCom), 2014 IEEE 13th International Conference on. IEEE, 2014. 
[6] Perera, Charith, et al. "Mosden: An internet of things middleware for resource constrained mobile devices." System Sciences (HICSS), 2014 47th Hawaii International Conference on. IEEE, 2014. 